Proceedings

\title{
DFT Study of the Decomposition Pathways of 5,5'-bitetrazole-1,1'-diide as a Parent Anion in the Family of Highly Energetic Green Explosives ${ }^{\dagger}$
}

\author{
Sergey V. Bondarchuk \\ Department of Chemistry and Nanomaterials Science, Bogdan Khmelnitsky Cherkasy National University; \\ Correspondence: bondchem@cdu.edu.ua; Tel.: +38-047-237-6576
}

Academic Editor: name

Received: date; Accepted: date; Published: date

\begin{abstract}
The decomposition pathways of the anionic parts of the 5,5'-bitetrazole-related energetic salts are studied theoretically using highly-symmetric 5,5'-bitetrazole-1,1'-diide (point group $\mathrm{D}_{2 \mathrm{~d}}$ ) as a model compound. The relaxed scans for all the four symmetry unique bonds elongation have been performed at the $w B 97 X \mathrm{D} / 6-311+G(d, p)$ level of theory. The results have revealed that the most preferable decomposition route is the $\mathrm{C} 5-\mathrm{N} 1$ bond elongation. Breaking of the bonds has been tracked using the QTAIM analysis at each point along the reaction coordinate. Another decomposition mechanism of 5,5'-bitetrazole-1,1'-diide can be triggered by its oxidation, which results in breaking of the $\mathrm{N} 2-\mathrm{N} 3$ bond.
\end{abstract}

Keywords: HEDM; nitrogen-rich explosive; energetic salt; DFT

\section{Introduction}

5,5'-Bitetrazole backbone is a building block for a number of highly effective explosive materials [1,2]. One of these explosives is dihydroxylammonium 5,5'-bistetrazole-1,10-diolate (TKX-50) [3]. The calculations of detonation profile for the latter revealed that TKX-50 exceeds one of the most powerful explosive, namely $\varepsilon$-hexanitrohexaazaisowurtzitane ( $\varepsilon$-CL-20) [3]. Moreover, impact sensitivity of TKX-50 (20 J) is lower than that of 2,4,6-trinitrotoluene (TNT) (15 J) [3]. In addition, the impact sensitivity of TKX-50 can be decreased by addition of polyvinylidene difluoride (PVDF) and polychlorotrifluoroe-thylene (PCTFE), forming the polymer-bonded explosives [4], or by cocrystallization with hexahydro-1,3,5-trinitro-1,3,5-triazine (RDX) [5]. The recent experimental studies, however, have shown that TKX-50 is close to RDX not only in terms of its sensitivity to impact but also in terms of other explosive parameters [6,7]. For example, the solid-state enthalpy of formation $\left(\Delta H_{298}^{0}\right.$ ) of TKX-50 based on the measurements of enthalpy of combustion equals $113.97 \pm$ $2.86 \mathrm{~kJ} \mathrm{~mol}^{-1}[6,7]$, whereas the calculated values using of the atomization method CBS-4M equals to $446.6 \mathrm{~kJ} \mathrm{~mol}^{-1}[3]$.

The decomposition mechanism of the TKX-50 crystals was extensively studied both experimentally and theoretically. To date, a two-step process initiated by a proton transfer from hydroxylammonium cations to 5,5'-bistetrazole-1,10-diolate is generally accepted [8-15]. Early molecular dynamics studies of thermal decomposition of TKX-50 revealed that the latter decays with release of molecular nitrogen or $\mathrm{N}_{2} \mathrm{O}$ with the activation energies of 188.7 and $302.1 \mathrm{~kJ} \mathrm{~mol}^{-1}$, respectively [8]. More recent experimental studies, however, give the activation energy value obtained by the Kissinger's method been equal to $237.6 \mathrm{~kJ} \mathrm{~mol}^{-1}$ [9]. The latter is strongly dependent on the experimental conditions (heating rate) and can be decreased even to $112 \mathrm{~kJ} \mathrm{~mol}^{-1}$ [10]. It is 
worthwhile to note that the solid substance bearing the ammonium cations instead of the hydroxylammonium cations was detected experimentally among the intermediate products of decomposition [10]. A number of intermediate structures were calculated as a result of the decomposition of electronically excited TKX-50 [11]. It was found that TKX-50 has two conical intersections between $S_{1}$ and $S_{0}$ states, related to and leading to two different reaction paths, forming $\mathrm{N}_{2}$ and NO products [11].

Upon applied external pressure and subsequent heating, the phase transition in the TKX-50 crystals occurs leading to two consecutive high-temperature intermediates $[12,13]$. This results in suppression of the proton transfer, and thus the impact sensitivity of TKX-50 [12,14]. It was also found that TKX-50 exhibits a strong anisotropy of impact sensitivity with [010] as the most sensitive direction and [100] as least sensitive [15].

Thus, in the present paper we have developed an approach for computational study the possible route of the bitetrazole backbone decomposition. For this purpose we have replaced the 5,5'-bistetrazole-1,10-diolate moiety by a more symmetric 5,5'-bitetrazole-1,1'-diide ion (BTEZ) and obtained relaxed scans of all possible routs of the bond breaking followed by the transition state search. As a result, we have obtained the activation energy equal $229.1 \mathrm{~kJ} \mathrm{~mol}^{-1}$, which is rather close to the Yang's value [9]. The formation of an azide anion was found to be the most exothermic path of the BTEZ decomposition.

\section{Computational Details}

The DFT calculations presented in this paper were performed using the GAUSSIAN09 program suite [16]. Geometry optimizations and vibrational spectra calculations were carried out using the range-separated hybrid functional parameterized by Chai and Head-Gordon, namely wB97XD [17]. The latter uses a version of the Grimme's D2 dispersion model, which is essential when calculating systems with strongly elongated bonds. The Pople's split-valence quasi triple- $\zeta$, in the valence shell, basis set $(6-311 \mathrm{G})$ with addition of both polarization $(\mathrm{d}, \mathrm{p})$ and diffuse $(+)$ functions was applied [18]. For the relaxed scans calculation, step size for the bond length change was set to $0.25 \AA$. Optimization of the transition states was performed using the quadratic synchronous transit method (QST3) [19].

Electronic spectra were calculated in terms of Time-Dependent DFT (TDDFT) in the vacuum approximation. The analysis of electron density distribution was carried out by means of the Quantum Theory of Atoms in Molecules (QTAIM) [20]. The calculations were performed using the AIMQB code as implemented in the AIMStudio program suite with the Proaim basin integration method [21]. Additional post-SCF analyses were conducted using the Multiwfn program package [22]. Fitting the electronic absorption spectrum curve was performed by means of the Gaussian distribution function and a half-width of $3000 \mathrm{~cm}^{-1}$ with the SWizard 5.0 program package [23].

\section{Results and Discussion}

Geometry optimization of BTEZ provides a structure of the $\mathrm{D}_{2 \mathrm{~d}}$ point group. The latter has the four symmetry non-equivalent bonds, namely C5-C5', C5-N1, N1-N2 and N2-N3. In order to find the most energetically preferable decomposition path, we have performed relaxed scan calculations, which are illustrated in Fig. 1 a. As it follows from Fig. 1 a, elongation of the C5-C5' and N2-N3 bonds leads to a gradual energy rise without maxima at least to $r_{0}+3.5$ and $r_{0}+2.25 \AA$, respectively. Meanwhile, stretching of the rest two bonds proceeds with maxima which are located at about $r_{0}+$ $0.75 \AA$ A (Fig. 1 a).

As a result, one of the three pairs of the decomposition products is expected to be formed (Fig. 1 b). The pair 1 corresponds to the $\mathrm{C} 5-\mathrm{N} 1$ bond breaking and the pairs 2 and 3 correspond to the $\mathrm{N} 1-\mathrm{N} 2$ bond cleavage. Total energy calculations for the species in pair 2 and 3 revealed that formation of the pair 2 is much more energetically preferable. Detachment of an azide radical from BTEZ is unlikely because of a huge endothermicity of the reaction $\left(+569.4 \mathrm{~kJ} \mathrm{~mol}^{-1}\right)$. The optimized structures of the three possible organic residues of BTEZ are illustrated in Fig. 2. 


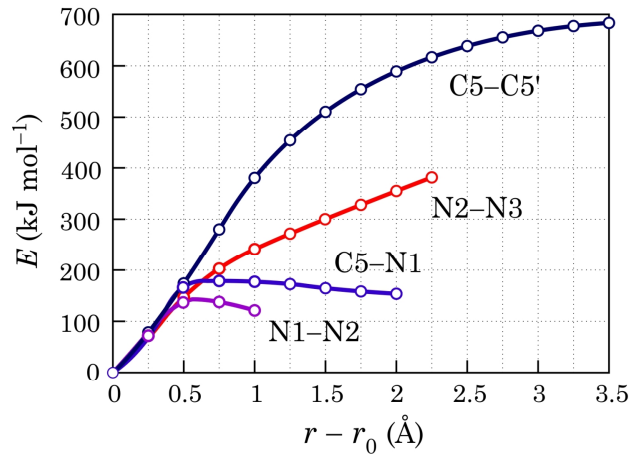

(a)

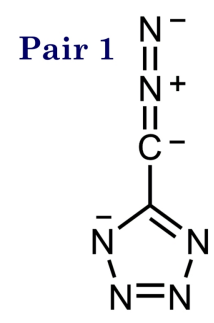

$\mathrm{N} \equiv \mathrm{N}$
Pair 2<smiles>N#Cc1nnn[nH]1</smiles>

$\overline{\mathrm{N}}=\stackrel{+}{\mathrm{N}}=\overline{\mathrm{N}}$

(b)

Figure 1. (a) The relaxed scans of the symmetry-unique bonds elongation in BTEZ; (b) the most relevant pairs of the decomposition products.

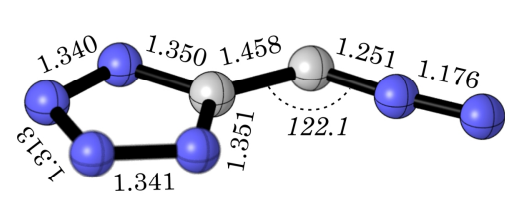

(a)

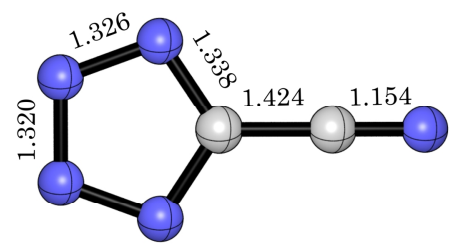

(b)

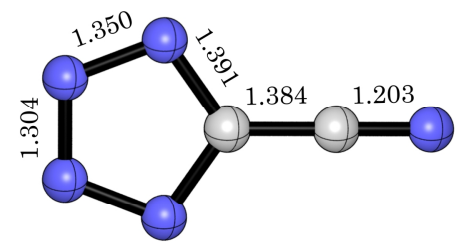

(c)

Figure 2. Optimized geometries and several structural parameters (angstroms and degrees) of: (a) 5-(diazomethideyl)tetrazol-1-ide; (b) 5-cyanotetrazol-1-ide; (c) 5-cyanotetrazol-1-ide radical anion.

Thus, we have checked two reaction paths leading to the pair 1 and 2. In order to localize the corresponding transition states (TS) we have applied QST3 method. The calculations provide close activation energies $c a .229 \mathrm{~kJ} \mathrm{~mol}^{-1}$ (Fig. 3 a). The reaction heat, however, differs significantly for these two reaction paths. Reaction proceeding through TS2 is endothermic $\left(\Delta H=+169.2 \mathrm{~kJ} \mathrm{~mol}^{-1}\right)$, while reaction route through TS1 is strongly exothermic $\left(\Delta H=-167.8 \mathrm{~kJ} \mathrm{~mol}^{-1}\right)$. The driving force of the latter reaction is stronger; therefore, it is anticipated to be more probable. Additionally, the absolute values of the first imaginary frequencies can provide information about the curvature of potential energy surface along the transition vectors that determines the TS region. Thus, TS1 has $v_{1}$ $=463.5 i \mathrm{~cm}^{-1}$, and TS2 has $v_{1}=478.0 i \mathrm{~cm}^{-1}$.

As one can see in Fig. 3 b, the C5-N1 bond breaking is a mandatory condition for BTEZ decomposition. In the case of TS1 an additional N1-N2 bond breaking takes place and in the case of TS2 this additional bond is N2-N3. The QTAIM analysis has shown that these elongated bonds still remain at the TSs points since the critical points of the $(3,-1)$ type were found. Also, the QTAIM charges prove that wave functions describe correctly these TSs, since the total charge on the leaving nitrogen atoms is close to zero (TS2) and -1 (TS1).

According to the Boltzmann distribution, thermal occupation of the energy level, which corresponds to such a huge activation barrier $\left(229 \mathrm{~kJ} \mathrm{~mol}^{-1}\right)$, can scarcely be achieved at temperatures lower than ca. $800 \mathrm{~K}$ [24]. It means that electron excitations can be involved into the mechanism of BTEZ decomposition. Thus, we have calculated electronic spectrum of BTEZ, which consists of first 20 transitions. Summary of the results of this calculation is presented in Table 1. As it follows from Table 1, a noticeable absorption starts from $S_{6}(5.90 \mathrm{eV})$. The $S_{1}$ state, however, lies much lower in energy $(5.12 \mathrm{eV})$. We have also optimized structure of the first excited triplet state $\left(T_{1}\right)$ and first excited singlet state $\left(S_{1}\right)$. Remarkably, both these states are planar ( $\mathrm{D}_{2 \mathrm{~h}}$ point group) meaning that $S_{0}$ $\rightarrow S_{1}$ transition is strongly nonadiabatic. The singlet state $S_{1}$ lies higher than the $T_{1}$ state by $0.77 \mathrm{eV}$ according to the adiabatic energy difference. 


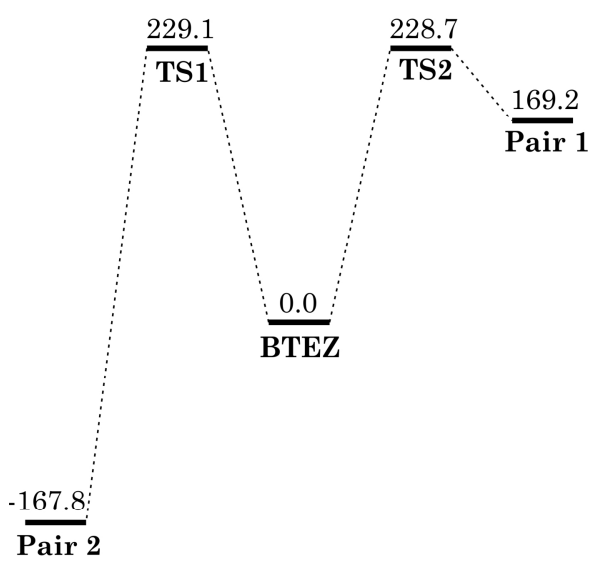

(a)


(b)

Figure 3. (a) Energy levels ( $\left.\mathrm{kJ} \mathrm{mol}^{-1}\right)$ of the reactants, TSs and products; (b) optimized geometries and several structural parameters of the calculated TSs.

Table 1. Assignment of the most intense $\left(f_{\text {osc }}>0.01\right)$ transitions in the electronic spectrum of BTEZ.

\begin{tabular}{ccccccc}
\hline State & $\lambda(\mathbf{n m})$ & $\boldsymbol{E} \mathbf{( e V )}$ & $f_{\text {osc }}$ & \multicolumn{3}{c}{ Orbital assignment } \\
\hline$S_{6}$ & 210.1 & 5.90 & 0.0795 & $\mathrm{H}^{\mathrm{a}-2} \rightarrow \mathrm{L}^{\mathrm{b}}(51 \%)$ & $\mathrm{H} \rightarrow \mathrm{L}+3(31 \%)$ & $\mathrm{H}-3 \rightarrow \mathrm{L}+1(13 \%)$ \\
$S_{8}$ & 208.7 & 5.94 & 0.0355 & $\mathrm{H}-1 \rightarrow \mathrm{L}+1(78 \%)$ & $\mathrm{H} \rightarrow \mathrm{L}+4(11 \%)$ & $\mathrm{H}-8 \rightarrow \mathrm{L}(7 \%)$ \\
$S_{9}$ & 206.4 & 6.01 & 0.0282 & $\mathrm{H}-4 \rightarrow \mathrm{L}(88 \%)$ & $\mathrm{H}-5 \rightarrow \mathrm{L}+1(9 \%)$ & \\
$S_{10}$ & 203.3 & 6.10 & 0.0609 & $\mathrm{H}-5 \rightarrow \mathrm{L}(54 \%)$ & $\mathrm{H}-4 \rightarrow \mathrm{L}+1(42 \%)$ & \\
$S_{11}$ & 202.7 & 6.12 & 0.0233 & $\mathrm{H}-6 \rightarrow \mathrm{L}(60 \%)$ & $\mathrm{H}-3 \rightarrow \mathrm{L}+1(19 \%)$ & $\mathrm{H}-7 \rightarrow \mathrm{L}+1(15 \%)$ \\
$S_{13}$ & 200.1 & 6.19 & 0.0218 & $\mathrm{H} \rightarrow \mathrm{L}+4(59 \%)$ & $\mathrm{H}-1 \rightarrow \mathrm{L}+6(11 \%)$ & $\mathrm{H}-1 \rightarrow \mathrm{L}+1(10 \%)$ \\
$S_{14}$ & 200.1 & 6.20 & 0.3262 & $\mathrm{H} \rightarrow \mathrm{L}+5(70 \%)$ & $\mathrm{H}-3 \rightarrow \mathrm{L}+1(10 \%)$ & $\mathrm{H}-4 \rightarrow \mathrm{L}+2(7 \%)$ \\
$S_{20}$ & 195.0 & 6.36 & 0.0184 & $\mathrm{H} \rightarrow \mathrm{L}+7(78 \%)$ & $\mathrm{H} \rightarrow \mathrm{L}+4(8 \%)$ & $\mathrm{H}-9 \rightarrow \mathrm{L}+1(5 \%)$ \\
\hline
\end{tabular}

${ }^{a} \mathrm{H}$ stands for the highest occupied molecular orbital; ${ }^{\mathrm{b}} \mathrm{L}$ stands for the lowest unoccupied molecular orbital.

Finally, when BTEZ is oxidized to the neutral state, the N2-N3 bonds significantly elongate, $1.580 \AA$ vs. $1.314 \AA$ (in dianion). Also, a strong aromatic character of the tetrazole rings in the dianion becomes slightly aromatic in the neutral form. This follows from the NICS (nucleus-independent chemical shift) indexes calculations, whereas the structural criteria, namely HOMA (harmonic oscillator model of aromaticity) and Bird, indicate the neutral form of BTEZ as antiaromatic. Thus, in the neutral form, the most probable route of decomposition is the detachment of a nitrogen molecule.

\section{Conclusions}

The obtained results revealed that the mechanism of BTEZ decomposition is not trivial. Huge activation barriers, obviously, should be lower when considering the ionic arrangement. Nevertheless, the activation barriers for real crystalline salts (like TKX-50 or MAD-X1) is expected to be high enough for a simple thermal occupation. This suggests the fairness of the previous results concerning conical intersections between $S_{1}$ and $S_{0}$ states [11].

Thus, a more detailed mechanistic study is mandatory to find the decomposition pathway, which includes all the experimentally detected intermediates. Particularly, recent results have shown that the TKX-50 decomposition includes a stage of diammonium 5,5'-bistetrazole-1,10-diolate 
(ABTOX) formation and also melamine-like solid polymer [10]. The account of all these circumstances will be the issue of further theoretical study.

Acknowledgments: This work was supported by the Ministry of Education and Science of Ukraine, Research Fund (Grant No. 0115U000637).

Conflicts of Interest: The author declares no conflict of interest.

\section{References}

1. Klapötke, T.M.; Kurz, M.Q.; Scharf, R.; Schmid, P.C.; Stierstorfer, J.; Sućeska, M. 5-(1H-Tetrazolyl)-2-Hydroxy-Tetrazole: A Selective 2N-Monoxidation of Bis(1H-Tetrazole). ChemPlusChem 2015, 80, 97-106, DOI: 10.1002/cplu.201402124.

2. Fischer, D.; Klapötke, T.M.; Reymann, M.; Schmid, P.C.; Stierstorfer, J.; Sućeska, M. Synthesis of 5-(1H-Tetrazolyl)-1-hydroxy-tetrazole and Energetically Relevant Nitrogen-Rich Ionic Derivatives. Prop. Explos. Pyrotech. 2010, 35, 1-8, DOI: 10.1002/prep.201300152.

3. Fischer, N.; Fischer, D.; Klapötke, T.M.; Piercey, D.G; Stierstorfer, J. Pushing the limits of energetic materials - the synthesis and characterization of dihydroxylammonium 5,5'-bistetrazole-1,10-diolate. J. Mater. Chem. 2012, 22, 20418-20422, DOI: 10.1039/c2jm33646d.

4. Ma, S.; Li, Y.; Li, Y.; Luo, Y. Research on structures, mechanical properties, and mechanical responses of TKX-50 and TKX-50 based PBX with molecular dynamics. J. Mol. Model. 2016, 22, 43, DOI: 10.1007/s00894-016-2913-4.

5. Xiong, S.; Chen, S.; Jin, S. Molecular dynamic simulations on TKX-50/RDX cocrystal. J. Mol. Graph. Model. 2017, 74, 171-176, DOI: 10.1016/j.jmgm.2017.03.006.

6. Sinditskii, V. P.; Filatov, S. A.; Kolesov, V. I.; Kapranov, K. O.; Suprun, A. O.; Asachenko, A. F.; Dzhevakov, P. B.; Topchiy, M. A.; Nechaev, M. S.; Lunin, V. V.; Shishov, N. I. Dihydroxylammonium 5,5'-bistetrazole-1,1'-diolate (TKX-50): breakthrough or an error? Combust. Explos. 2015, 8, 186-194.

7. Sinditskii, V.P.; Filatov, S.A.; Kolesov, V.I.; Kapranov, K.O.; Asachenko, A.F.; Nechaev, M.S.; Lunin, V.V.; Shishov, N.I. Combustion behavior and physico-chemical properties of dihydroxylammonium 5,5'-bistetrazole-1,1'-diolate (TKX-50). Thermochim. Acta 2015, 614, 85-92, DOI: 10.1016/j.tca.2015.06.019.

8. An, Q.; Liu, Q.-G.; Goddard III, W.A.; Cheng, T.; Zybin, S.V.; Xiao, H. Initial Steps of Thermal Decomposition of Dihydroxylammonium 5,5'-bistetrazole-1,1'-diolate Crystals from Quantum Mechanics. J. Phys. Chem. C 2014, 118, 27175-27181, DOI: 10.1021/jp509582x.

9. Huang, H.; Shi, Y.; Yang, J. Thermal characterization of the promising energetic material TKX-50, J. Therm. Anal. Calorim. 2015, 121, 705-709, DOI 10.1007/s10973-015-4472-9.

10. Muravyev, N.V.; Monogarov, K.A.; Asachenko, A.F.; Nechaev, M.S.; Ananyev, I.V.; Fomenkov, I.V.; Kiselev, V.G.; Pivkina, A.N. Pursuing reliable thermal analysis techniques for energetic materials: decomposition kinetics and thermal stability of dihydroxylammonium 5,5'-bistetrazole-1,10-diolate (TKX-50). Phys. Chem. Chem. Phys. 2017, 19, 436-449, DOI: 10.1039/c6cp06498a.

11. Yuan, B.; Yu, Z.; Bernstein, E.R. Initial Mechanisms for the Decomposition of Electronically Excited Energetic Salts: TKX-50 and MAD-X1. J. Phys. Chem. A 2015, 119, 2965-2981, DOI: 10.1021/jp510995z.

12. Dreger, Z.A.; Breshike, C.J;; Gupta, Y.M. High pressure - high temperature phase diagram of an energetic crystal: dihydroxylammonium 5,5'-bistetrazole-1,1'-diolate (TKX-50). Chem. Phys. Lett. 2017, 679, 212-218, DOI: 10.1016/j.cplett.2017.05.019.

13. Lu, Z.; Xue, X.; Meng, L.; Zeng, Q.; Chi, Y.; Fan, G.; Li, H.; Zhang, Z.; Nie, F.; Zhang, C. Heat-Induced Solid-Solid Phase Transformation of TKX-50. J. Phys. Chem. C 2017, 121, 8262-8271, DOI: 10.1021/acs.jpcc.7b00086.

14. Lu, Z.; Zhang, C. Reversibility of the Hydrogen Transfer in TKX-50 and Its Influence on Impact Sensitivity: An Exceptional Case from Common Energetic Materials. J. Phys. Chem. C 2017, 121, 21252-21261, DOI: 10.1021/acs.jpcc.7b07663.

15. An, Q.; Cheng, T.; Goddard III, W.A.; Zybin, S.V. Anisotropic Impact Sensitivity and Shock Induced Plasticity of TKX-50 (Dihydroxylammonium 5,5'-bis(tetrazole)-1,1'-diolate) Single Crystals: From Large-Scale Molecular Dynamics Simulations. J. Phys. Chem. C 2015, 119, 2196-2207, DOI: 10.1021/jp510951s. 
16. Frisch, M. J.; Trucks, G. W.; Schlegel, H. B.; Scuseria, G. E.; Robb, M. A.; Cheeseman, J. R.; Scalmani, G.; Barone, V.; Mennucci, B.; Petersson, G. A.; et al. Gaussian 09, Revision A.02; Gaussian, Inc.: Wallingford, CT, 2009. Available at www.gaussian.com.

17. Chai, J.-D.; Head-Gordon, M. Long-range corrected hybrid density functionals with damped atom-atom dispersion corrections. Phys. Chem. Chem. Phys. 2008, 10, 6615-6620, DOI: 10.1039/b810189b.

18. Hehre, W. J.; Radom, L.; Schleyer, P. v. R.; Pople, J. A. Ab Initio Molecular Orbital Theory, Wiley, New York, 1986.

19. Peng, C.; Schlegel, H. B. Combining Synchronous Transit and Quasi-Newton Methods to Find Transition States. Israel J. Chem. 1993, 33, 449-454, DOI: 10.1002/ijch.199300051.

20. Bader, R. F. W. Atoms in molecules. A quantum theory; Clarendon Press: Oxford, U.K., 1990.

21. Keith, T. A. AIMAll, Version 10.07.25; TK Gristmill Software: Overland Park, KS, 2010. Available at www.aim.tkgristmill.com.

22. Lu, T.; Chen, F. Multiwfn: A Multifunctional Wavefunction Analyzer. J. Comput. Chem. 2012, 33, 580-592, DOI: $10.1002 /$ jcc. 22885 .

23. Gorelsky, S. I. SWizard program; University of Ottawa: Ottawa, Canada, 2013; Available at www.sg-chem.net.

24. Bondarchuk, S. V.; Minaev, B. F. Thermally accessible triplet state of $\pi$-nucleophiles does exist. Evidence from first principles study of ethylene interaction with copper species. RSC Adv. 2015, 5, 11558-11569, DOI: $10.1039 / \mathrm{c} 4 \mathrm{ra12422g}$.

(C) 2017 by the authors. Submitted for possible open access publication under the terms and conditions of the Creative Commons Attribution (CC BY) license (http://creativecommons.org/licenses/by/4.0/). 